\title{
Study of Hand and Finger Indices for Prediction of Sex and Estimation of Stature in a Sample of Egyptian and Malaysian Youth
}

\author{
Amal S. A. F. Hafez ${ }^{* 1}$, MD, Marwa M. Shahin, MD
}

\begin{tabular}{ll}
\hline & ABSTRACT \\
\cline { 2 - 3 } KEYWORDS & \multicolumn{1}{c}{ Identification is a cornerstone in forensic investigations. The present study } \\
Hand measurements; & aimed to evaluate hand indices as a potential tool for determination of sex and \\
Index finger length; & estimation of stature in a sample of Egyptian and Malaysian people. The study \\
Ring finger length; & included 200 Egyptian and 100 Malaysian participants. Stature was measured \\
D2:D4 ratio; & using a stadiometer. Hand length, hand breadth, index finger length, and ring \\
Prediction of sex; & finger length were measured using Vernier caliper. The hand index was computed \\
Estimation of stature. & by the following formula: (hand breadth/hand length) 100 . The ratio of the index \\
& to the ring finger (D2:D4 ratio) was calculated by dividing the length of index by \\
the length of the ring finger of the same hand. All measurements were significantly & higher in male than female subjects, except for the D2:D4 ratio which was \\
& significantly higher in female than in male subjects in either population. In \\
& Egyptian participants, the best measurement for sex discrimination was hand \\
length in both hands (AUC: Rt=0.892, Lt: AUC=0.898). In Malaysian participants, \\
the best measurement for sex discrimination was hand breadth in both hands (Rt: \\
AUC=0.949, Lt: AUC=0.959). In all groups, there was positive, significant, \\
moderate-strong correlation between stature and each of hand length, hand \\
breadth, index finger length, and ring finger length. Backward elimination \\
regression was performed to develop models for prediction of sex and estimation of \\
stature for each population using measurements from one hand. In conclusion, \\
hand dimensions can serve as a tool to predict sex and calculate stature with \\
reasonable accuracy in Egyptian and Malaysian youths.
\end{tabular}

\section{Introduction}

Identification is a cornerstone in forensic investigations. It is common to find dismembered human remains and peripheral parts of the body in incidents like mass disasters, either natural or in cases of war, bombing and traffic accidents or acts of terrorism (Aboul-Hagag et al., 2011).

\footnotetext{
(I) Forensic Medicine and Clinical Toxicology Department Tanta Faculty of Medicine, Tanta University, Tanta, Egypt.

*Corresponding Author: Amal S. A. F. Hafez, e-mail: amal.hafez@med.tanta.edu.eg
}

Forensic anthropometry is a scientific specialization out of forensic anthropology using metric techniques for purpose of identification of human remains (Krishan, 2007).

Many researchers believe that different sex hormone exposure during early embryonic development "estrogen and testosterone concentrations in utero", which is controlled by HOX genes, results in relative difference in lengths of fingers in human population, and this area of research still attract a considerable research interest (Ventura et al., 2013; Warrington et al., 2018). The Hox genes 
belong to the group of homeobox genes which specify the characteristics of structures in anatomical regions (patterning of body) (Hrycaj and Wellik, 2016).

Determining sex of the remains narrows down the pool of possible victim matches as it excludes half the population (Swami et al., 2013). Prediction of stature occupies an important position both in the medicolegal identification and in anthropological research (Jasuja and Singh, 2004). There is a wellknown relationship between stature and measurements of various body parts like upper and lower extremities as well as head and trunk (Krishan and Sharma, 2007).

Many studies have proposed regression equations for estimation of stature, and they are accurate, reliable and of utmost importance for solving problems in medico-legal cases by forensic scientists. However, bone measurements and characteristics were found to differ between various human races (Looker et al., 2009). Therefore, there is a need to conduct studies that offer specific sexing parameters and stature equations for each population. Some previous studies have evaluated hand measurements as a tool to estimate stature and identify sex, both in Egypt (Habib and Kamal, 2010; Aboul-Hagag et al., 2011) and other countries (Varu et al., 2015; Jeyaseelan et al., 2016; Camacho-Hernández et al., 2018; Rhiu and Kim, 2019).

The aim of the present study was to evaluate hand indices as a potential tool for determination of sex and estimation of stature in a sample of Egyptian and Malaysian people.

\section{Material and Methods}

\section{Study settings}

This cross-sectional study was carried out from start of December 2016 till the end of May 2017 on 300 healthy Egyptian and Malaysian participants at Forensic Medicine and Clinical Toxicology department, Faculty of Medicine, Tanta University.

\section{Ethical considerations}

The study was carried out after obtaining ethical approval from the Research Ethics Committee, Faculty of Medicine, Tanta University. A written informed consent was taken from every subject before participating in the study. Confidentiality was maintained by assigning a code number for every participant, and the codes were known only by the investigators.

\section{Study population}

The study sample included 300 volunteers (200 Egyptians and 100 Malaysians) whose age was above 21 and below 30 years old.

\section{Inclusion criteria:}

Egyptian and Malaysian volunteers of either sex, who was above 21 and below 30 years old, were included in this study.

\section{Exclusion criteria:}

Volunteers with any of the following conditions were excluded from the study: presence of any physical deformity, injury, fracture, or amputation as well as history of surgical procedures involving the limbs.

\section{Data collection:}

All participants were subjected to history taking, clinical examination, and taking of measurements. History taking included personal data (age, sex, and nationality) and history of any trauma, disease or surgery involving the limbs. Clinical examination was conducted with the aim of identifying any abnormality affecting the stature or upper limbs.

The stature was measured using as vertical distance from the vertex to the foot by 
a stadiometer. Each participant was asked to stand on a horizontal resting plane on the stadiometer. The participants were instructed to stand erect, the head in eye-ear plane, their feet were bare, and the palms of their hands turned inwards and the fingers vertically pointing downwards (Frankfurt plane).

Hand measurements included hand length, hand breadth, index finger length, and ring finger length. The measurements were taken for both right and left hands using a Vernier caliper and following the methods described by Weiner and Laurie (1969). During measurement, the upper limb was placed so as the forearm was directly in line with the middle finger. The hand length was measured starting from the distal crease of wrist joint up to the tip of middle finger. The hand breadth was measured starting from the most laterally placed point on the head of the second metacarpal bone up to the most medially placed point on the head of the fifth metacarpal bone. The lengths of index and ring fingers were measured from the tip of the finger to the metacarpophalangeal crease (Figure 1). All measurements were obtained by one observer to avoid inter-observer variability.

The hand index was computed by the following formula: (hand breadth/hand length) $x 100$. The ratio of the index to the ring finger (D2:D4 ratio) in each hand was calculated by dividing the length of index by the length of the ring finger of the same hand.

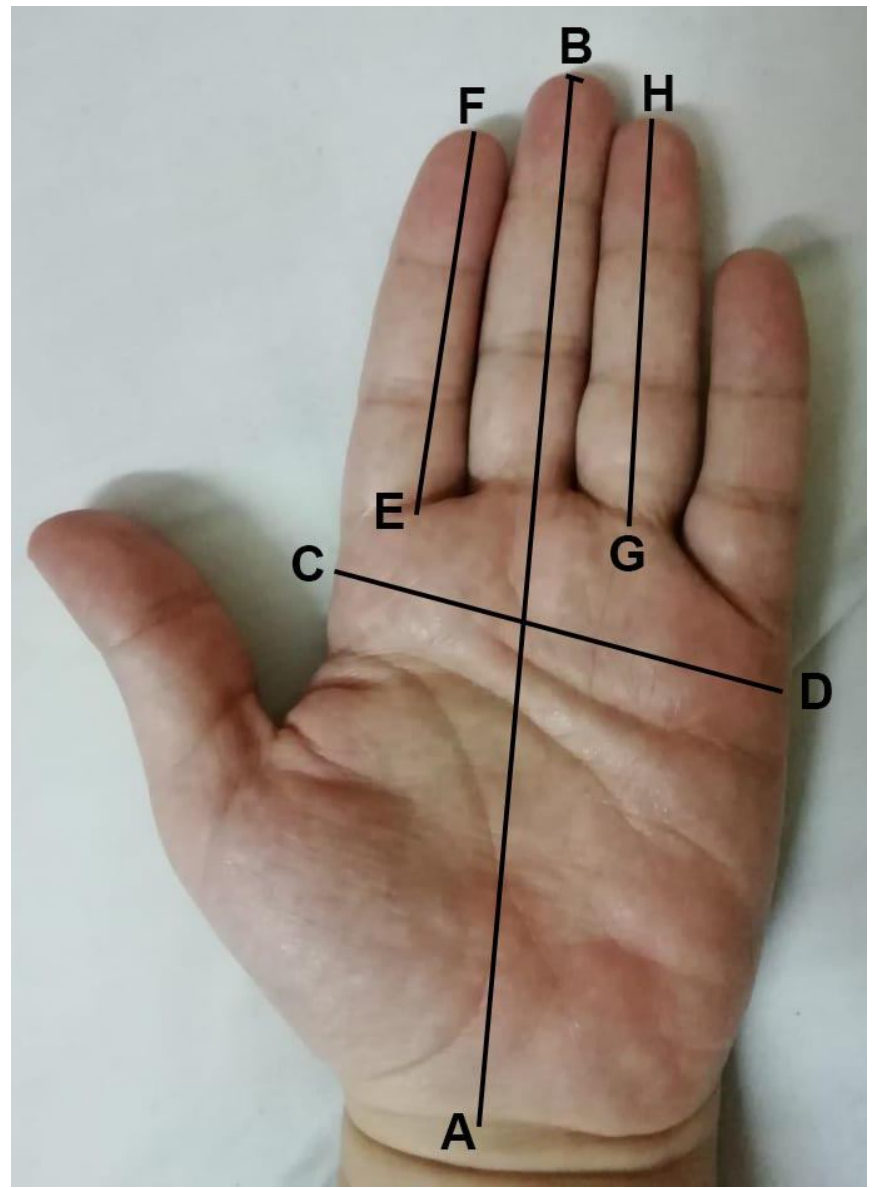

Fig. (1): Human hand illustrating the landmarks of hand length $(A: B)$, hand breadth $(C: D)$, index finger length $(\mathrm{E}: \mathrm{F})$, and ring finger length $(\mathrm{G}: \mathrm{H})$. 


\section{Statistical analysis:}

Statistical analysis was performed using MedCalc Statistical Software version 15.8 (MedCalc Software bvba, Ostend, Belgium; https://www.medcalc.org; 2015). The Shapiro-Wilk test for normality besides visual assessment of graphs were performed, revealing normal distribution of the studied numerical variables. Numerical variables were summarized as mean \pm standard deviation (SD) and range (minimummaximum). Comparison between unpaired two groups was performed using Independent samples T-test, while paired T-test was used to compare measurements between right and left hands and fingers. Analysis of receiver operating characteristic (ROC) curve was done to assess discriminatory power of sex for each measurement according to the method by DeLong et al. (1988). The area under ROC curve was graded as follows: 0.90-1 = excellent; $0.80-0.90=$ good; $0.70-0.80=$ fair; and $0.60-0.70=$ poor. Sensitivity and specificity were calculated. Sensitivity represented the percentage of those identified by a measurement as men who belonged really to male sex. Specificity represented the percentage of those identified by a measurement as women who really were female. Spearman's rank-order correlation was performed to examine direction and strength of relationship between stature and hand and finger measurements. Backward, stepwise logistic regression was performed to identify significant measurements for prediction of male sex. Backward, stepwise multiple regression was conducted to identify significant measurements for estimation of stature. A p-value $<0.05$ was adopted for interpretation of statistical tests.

\section{Results}

\section{Characteristics of participants}

The present study included 300 participants: 200 Egyptians (103 female and 97 male participants) and 100 Malaysians (50 female and 50 male participants). Table (1) summarizes the characteristics of participants. The mean age was comparable among the male and female subjects of both populations. The mean stature was higher in Egyptian male participants $(174.9 \pm 7.2 \mathrm{~cm})$, followed by Malaysian males $(169.1 \pm 6.3 \mathrm{~cm})$, Egyptian females $(161.7 \pm 5.2 \mathrm{~cm})$, and lastly Malaysian female subjects $(158.8 \pm 5.8 \mathrm{~cm})$.

\section{Comparison of measurements between right and left hands and fingers}

Paired T-test was used to compare between measurements of the right and left hands and fingers of the studied subjects. The test revealed the lack of significant difference between right- and left-side measurements within all categories of participants $(p>0.05)$.

\section{Comparison of measurements between male and female subjects}

Tables (2) and (3) compared the measurements of both right and left hands and fingers between the studied male and female subjects. All measurements were significantly higher in male than female subjects (regardless of population), except for the D2:D4 ratio which was significantly higher in female than in male subjects in either population.

\section{Determination of sex using hand and finger measurements}

The discriminatory power for identification of sex of the individual studied hand measurements was evaluated using ROC curve analysis and determination of the AUC. 
Figures $(2-5)$ demonstrate for each measurement the AUC, the optimal cut-off point, as well as the associated sensitivity and specificity for the cut-off point.

In Egyptian participants, the best measurement for sex discrimination was hand length in both hands (AUC: Rt=0.892, Lt: $\mathrm{AUC}=0.898$ ), followed by ring finger length (Rt: $\mathrm{AUC}=0.875$, Lt: $\mathrm{AUC}=0.872$ ), hand breadth (Rt: $\mathrm{AUC}=0.852$, Lt: $\mathrm{AUC}=0.862$ ), length of index finger (Rt: $\mathrm{AUC}=0.834, \mathrm{Lt}$ : $\mathrm{AUC}=0.839), \mathrm{D} 2: \mathrm{D} 4$ ratio (Rt: $\mathrm{AUC}=0.649$, Lt: $\mathrm{AUC}=0.651$ ), and hand index (Rt: $\mathrm{AUC}=0.595$, Lt: $\mathrm{AUC}=0.602$ ).

As for Malaysian participants, the best measurement for sex discrimination was hand breadth in both hands (Rt: $\mathrm{AUC}=0.949, \mathrm{Lt}$ : $\mathrm{AUC}=0.959$ ), followed by ring finger length (Rt: $\mathrm{AUC}=0.938$, Lt: $\mathrm{AUC}=0.945$ ), hand length (Rt: $\mathrm{AUC}=0.915, \mathrm{Lt}=0.916$ ), length of index finger (Rt: $\mathrm{AUC}=0.880$, $\mathrm{Lt}$ : $\mathrm{AUC}=0.899$ ), $\mathrm{D} 2: \mathrm{D} 4$ ratio (Rt: $\mathrm{AUC}=0.763$, $\mathrm{Lt}: \mathrm{AUC}=0.762)$ and hand index (Rt: $\mathrm{AUC}=0.726, \mathrm{Lt}: \mathrm{AUC}=0.734)$.

The use of multiple measurements for prediction of sex was also attempted by performing regression analysis. Table (4) shows the results of backward elimination, logistic regression analysis for prediction of male sex using hand and finger measurements. A model was developed for each population using measurements from one hand to simulate some real-world situations when one amputated hand only may be found and sent for forensic examination. Therefore, four models were developed (right and left hands in Egyptians; right and left hands in Malaysians). All measurements of one side were entered into the analysis, then the statistical software excluded measurements which did not contribute significantly to prediction of sex. The final models are shown in table (4) and they include measurements which significantly contributed to prediction of sex (indicated by a $p$ value $<0.05$ ) and other measurements which considerably affected the $\mathrm{R}$ square value of the model if removed though their $p$-value was $>0.05$. The percentage of variation which is explained by the developed models was represented by Nagelkerke $\mathrm{R}$ square and was acceptable in the four models.

The equations for prediction of sex in Egyptians were as follows:

From right hand

Logit $(\mathrm{p}$ for male sex $)=-8.50+0.50 \mathrm{x} \mathrm{Rt}$ hand breadth $(\mathrm{mm})-0.71 \mathrm{x}$ Rt hand index (mm)

From left hand

Logit $(\mathrm{p}$ for male sex $)=148.88+0.44 \mathrm{x}$ Lt hand breadth $(\mathrm{mm})+2.22 \mathrm{x} \mathrm{Lt}$ index length $(\mathrm{mm})-2.13 \mathrm{x}$ Lt ring length $(\mathrm{mm})-0.61 \mathrm{x} \mathrm{Lt}$ hand index - 164.10 x Lt D2:D4 ratio

The equations for prediction of sex in Malaysians were as follows:

From right hand

Logit $(\mathrm{p}$ for male sex $)=-120.51+0.58 \mathrm{x}$ Rt hand length $(\mathrm{mm})-0.73 \mathrm{x}$ Rt hand breadth $(\mathrm{mm})-0.47 \mathrm{x}$ Rt index length $(\mathrm{mm})+0.49 \mathrm{x}$ $\mathrm{Rt}$ ring length $(\mathrm{mm})+1.65 \mathrm{x}$ Rt hand index

From left hand

Logit $(p$ for male sex $)=-88.58+0.26 x$ Lt hand length $(\mathrm{mm})-0.37 \mathrm{x}$ Lt index length $(\mathrm{mm})+0.57 \mathrm{x}$ Lt ring length $+0.65 \mathrm{x}$ Lt hand index

All derived equations estimated logit(p). To estimate $p$ (probability of being male), $\operatorname{logit}(p)$ is transformed by the following formula: $p=\frac{1}{1+e^{-\operatorname{logit}(p)}}$

\section{Correlation of stature with hand and finger measurements}

Table (5) illustrates the direction and strength of correlation between the stature and 
the studied hand and finger measurements in each category of participants. In all groups, there was positive, significant, moderate to strong correlation between stature and each of hand length, hand breadth, index finger length, and ring finger length. However, the correlation between stature and each of hand index and D2:D4 ratio was weak $(r<0.3$ or $>$ 0.3 ) and non-significant $(\mathrm{p}>0.05)$.

\section{Estimation of stature from hand and finger measurements}

Table (6) displays the results of backward elimination, multiple regression analysis for estimation of stature from hand and finger measurements. A model was developed for each population using measurements from one hand and four models were developed. All measurements of one side were entered into the analysis, then the statistical software excluded measurements which did not contribute significantly to stature estimation. The final models are shown in table (6) and they include measurements which significantly contributed to stature estimated (indicated by a $p$ value $<0.05)$ and other measurements which considerably affected the $\mathrm{R}$ square value of the model if removed though their $p$-value was $>0.05$. The percentage of variation which is explained by the developed models was represented by adjusted $\mathrm{R}$ square and was acceptable in the four models.

Equations for estimations of stature were derived using the constant and regression coefficients (B) for included measurements.

The equations for stature estimation in Egyptians were as follows:

From right hand

Stature $(\mathrm{cm})=79.02+0.38 \mathrm{x}$ Rt hand length $(\mathrm{mm})+0.24 \times$ Rt ring finger length $(\mathrm{mm})+5.93 \times \mathrm{Sex}$

From left hand

Stature $(\mathrm{cm})=71.93+0.52 \mathrm{x} \mathrm{Lt}$ hand length $(\mathrm{mm})+5.60 \times$ Sex

The equations for stature estimation in Malaysians were as follows:

From right hand

Stature $(\mathrm{cm})=-56.6+1.18 \mathrm{x}$ Rt hand length $(\mathrm{mm})-1.82 \times$ Rt hand breadth $(\mathrm{mm})+$ $3.46 \times$ Rt hand index $+3.30 \times$ Sex

From left hand

Stature $(\mathrm{cm})=-71.87+1.30 \mathrm{x} \mathrm{Lt}$ hand length $(\mathrm{mm})-2.12 \times$ Lt hand breadth $(\mathrm{mm})+$ $3.87 \mathrm{x}$ Lt hand index $+3.43 \mathrm{x}$ Sex

Sex was coded as 0 if female and 1 if male.

Table (1): Characteristics of participants:

\begin{tabular}{|l|c|c|c|c|}
\hline \multirow{2}{*}{} & \multicolumn{4}{|c|}{ Mean \pm SD (Range) } \\
\cline { 2 - 5 } & \multicolumn{2}{|c|}{ Egyptian } & \multicolumn{2}{c|}{ Malaysian } \\
\cline { 2 - 5 } & $\begin{array}{c}\text { Male } \\
(\mathbf{n}=\mathbf{9 7})\end{array}$ & $\begin{array}{c}\text { Female } \\
(\mathbf{n}=\mathbf{1 0 3})\end{array}$ & $\begin{array}{c}\text { Male } \\
(\mathbf{n}=\mathbf{5 0})\end{array}$ & $\begin{array}{c}\text { Female } \\
(\mathbf{n}=\mathbf{5 0})\end{array}$ \\
\hline \multirow{2}{*}{ Age (years) } & $22.4 \pm 1.7$ & $22.9 \pm 2.1$ & $23.8 \pm 1.6$ & $22.5 \pm 1.5$ \\
& $(21.0-30.0)$ & $(21.0-30.0)$ & $(21.0-27.0)$ & $(21.0-28.0)$ \\
\hline \multirow{2}{*}{ Stature $(\mathrm{Cm})$} & $174.9 \pm 7.2$ & $161.7 \pm 5.2$ & $169.1 \pm 6.3$ & $158.8 \pm 5.8$ \\
& $(161.0-190.0)$ & $(150.0-177.0)$ & $(157.0-184.0)$ & $(148.0-174.0)$ \\
\hline
\end{tabular}

SD: standard deviation. 
Table (2): Comparison of right hand and fingers measurements between men and women in the Egyptian and Malaysian participants:

\begin{tabular}{|c|c|c|c|c|c|c|}
\hline & \multicolumn{6}{|c|}{ Measurements Mean \pm SD (range) } \\
\hline & \multicolumn{3}{|c|}{ Egyptian } & \multicolumn{3}{|c|}{ Malaysian } \\
\hline & $\begin{array}{c}\text { Male } \\
(n=97)\end{array}$ & $\begin{array}{c}\text { Female } \\
(n=103)\end{array}$ & $\mathbf{p}^{\mathbf{a}}$ & $\begin{array}{c}\text { Male } \\
(n=50)\end{array}$ & $\begin{array}{l}\text { Female } \\
(n=50)\end{array}$ & $\mathbf{p}^{a}$ \\
\hline $\begin{array}{l}\text { Rt hand length } \\
(\mathrm{mm})\end{array}$ & $\begin{array}{c}188.3 \pm 9.0(170.5 \\
-206.1) \\
\end{array}$ & $\begin{array}{c}173.9 \pm 7.7 \\
(157.8-194.3) \\
\end{array}$ & $<0.001 * * *$ & $\begin{array}{c}182.8 \pm 12.5 \\
(120.9-197.5) \\
\end{array}$ & $\begin{array}{c}165.7 \pm 7.7 \\
(151.0-181.5) \\
\end{array}$ & $<0.001 * * *$ \\
\hline $\begin{array}{l}\text { Rt hand breadth } \\
(\mathrm{mm})\end{array}$ & $\begin{array}{c}84.9 \pm 5.6 \\
(72.2-95.9) \\
\end{array}$ & $\begin{array}{c}77.1 \pm 4.6 \\
(68.9-89.1) \\
\end{array}$ & $<0.001 * * *$ & $\begin{array}{c}82.5 \pm 4.5 \\
(70.8 \pm 90.5) \\
\end{array}$ & $\begin{array}{c}71.9 \pm 4.0 \\
(64.8 \pm 87.2) \\
\end{array}$ & $<0.001 * * *$ \\
\hline $\begin{array}{l}\text { Rt index length } \\
(\mathrm{mm})\end{array}$ & $\begin{array}{c}73.8 \pm 5.0 \\
(65.7-88.0) \\
\end{array}$ & $\begin{array}{c}67.7 \pm 3.7 \\
(59.7-78.8)\end{array}$ & $<0.001 * * *$ & $\begin{array}{c}72.0 \pm 4.5 \\
(62.4-81.2) \\
\end{array}$ & $\begin{array}{c}65.0 \pm 3.9 \\
(59.1-77.3) \\
\end{array}$ & $<0.001 * * *$ \\
\hline $\begin{array}{l}\text { Rt ring length } \\
(\mathrm{mm})\end{array}$ & $\begin{array}{c}75.6 \pm 4.9 \\
(66.0-88.9) \\
\end{array}$ & $\begin{array}{c}68.2 \pm 4.2 \\
(58.0-80.7) \\
\end{array}$ & $<0.001 * * *$ & $\begin{array}{c}74.0 \pm 4.6 \\
(65.1-82.2) \\
\end{array}$ & $\begin{array}{c}64.8 \pm 4.0 \\
(56.7-78.6) \\
\end{array}$ & $<0.001 * * *$ \\
\hline Rt hand index & $\begin{array}{c}45.1 \pm 2.2 \\
(39.0-51.0) \\
\end{array}$ & $\begin{array}{c}44.3 \pm 2.3 \\
(38.7-49.5) \\
\end{array}$ & $0.022 *$ & $\begin{array}{c}45.3 \pm 3.9 \\
(39.0-68.0) \\
\end{array}$ & $\begin{array}{c}43.4 \pm 2.0 \\
(40.3-51.8) \\
\end{array}$ & $0.003 * *$ \\
\hline Rt D2:D4 ratio & $\begin{array}{c}0.98 \pm 0.03 \\
(0.87-1.04)\end{array}$ & $\begin{array}{c}0.99 \pm 0.04 \\
(0.88-1.07)\end{array}$ & $0.001 * *$ & $\begin{array}{c}0.97 \pm 0.03 \\
(0.91-1.07)\end{array}$ & $\begin{array}{c}1.00 \pm 0.03 \\
(0.95-1.09)\end{array}$ & $<0.001 * * *$ \\
\hline
\end{tabular}

a: $\mathrm{p}$-value was calculated from independent samples T-test; SD: standard deviation; ${ }^{*} \mathrm{p}<0.05 ; * * \mathrm{p}<0.01, * * * \mathrm{p}<0.001$

Table (3): Comparison of left hand and fingers measurements between men and women in the Egyptian and Malaysian participants

\begin{tabular}{|c|c|c|c|c|c|c|}
\hline & \multicolumn{6}{|c|}{ Measurements $\quad$ Mean \pm SD (range) } \\
\hline & \multicolumn{3}{|c|}{ Egyptian } & \multicolumn{3}{|c|}{ Malaysian } \\
\hline & $\begin{array}{c}\text { Male } \\
(\mathrm{n}=97) \\
\end{array}$ & $\begin{array}{c}\begin{array}{c}\text { Female } \\
(n=103)\end{array} \\
\end{array}$ & $\mathbf{p}^{\mathbf{a}}$ & $\begin{array}{c}\text { Male } \\
(n=50) \\
\end{array}$ & $\begin{array}{c}\text { Female } \\
(\mathrm{n}=50) \\
\end{array}$ & $\mathbf{p}^{\mathbf{a}}$ \\
\hline $\begin{array}{l}\text { Lt hand length } \\
(\mathrm{mm})\end{array}$ & $\begin{array}{c}188.7 \pm 8.8 \\
(167.1-205.2) \\
\end{array}$ & $\begin{array}{c}174.0 \pm 7.7 \\
(155.0-194.6) \\
\end{array}$ & $<0.001 * * *$ & $\begin{array}{c}183.4 \pm 12.6 \\
(121.1-198.5) \\
\end{array}$ & $\begin{array}{c}166.2 \pm 7.5 \\
(151.8-183.0) \\
\end{array}$ & $<0.001 * * *$ \\
\hline $\begin{array}{l}\text { Lt hand breadth } \\
(\mathrm{mm})\end{array}$ & $\begin{array}{c}84.5 \pm 5.3 \\
(71.9-93.4) \\
\end{array}$ & $\begin{array}{c}76.5 \pm 4.9 \\
(66.9-89.8) \\
\end{array}$ & $<0.001 * * *$ & $\begin{array}{c}82.2 \pm 4.4 \\
(73.5-92.3) \\
\end{array}$ & $\begin{array}{c}71.0 \pm 4.8 \\
(60.9-84.5) \\
\end{array}$ & $<0.001 * * *$ \\
\hline $\begin{array}{l}\text { Lt index length } \\
(\mathrm{mm})\end{array}$ & $\begin{array}{c}74.0 \pm 4.9 \\
(65.8-88.3) \\
\end{array}$ & $\begin{array}{c}67.8 \pm 3.7 \\
(60.1-78.4) \\
\end{array}$ & $<0.001 * * *$ & $\begin{array}{c}72.2 \pm 4.2 \\
(63.2-81.1) \\
\end{array}$ & $\begin{array}{c}64.6 \pm 4.1 \\
(57.5-76.1) \\
\end{array}$ & $<0.001 * * *$ \\
\hline $\begin{array}{l}\text { Lt ring length } \\
(\mathrm{mm})\end{array}$ & $\begin{array}{c}75.8 \pm 4.9 \\
(64.6-89.2) \\
\end{array}$ & $\begin{array}{c}68.3 \pm 4.4 \\
(58.7-81.7) \\
\end{array}$ & $<0.001 * * *$ & $\begin{array}{c}74.2 \pm 4.5 \\
(66.4-82.5) \\
\end{array}$ & $\begin{array}{c}64.4 \pm 4.4 \\
(56.8-77.4) \\
\end{array}$ & $<0.001 * * *$ \\
\hline Lt hand index & $\begin{array}{c}44.8 \pm 2.1 \\
(40.5-49.8) \\
\end{array}$ & $\begin{array}{c}44.0 \pm 2.4 \\
(38.3-49.9) \\
\end{array}$ & $0.016^{*}$ & $\begin{array}{c}45.0 \pm 3.8 \\
(40.3-68.0) \\
\end{array}$ & $\begin{array}{c}42.7 \pm 2.5 \\
(36.6-49.0) \\
\end{array}$ & $0.001 * *$ \\
\hline Lt D2:D4 ratio & $\begin{array}{c}0.98 \pm 0.03 \\
(0.87-1.04) \\
\end{array}$ & $\begin{array}{c}0.99 \pm 0.04 \\
(0.88-1.08) \\
\end{array}$ & $<0.001 * * *$ & $\begin{array}{c}0.97 \pm 0.03 \\
(0.91-1.08) \\
\end{array}$ & $\begin{array}{r}1.00 \pm 0.03 \\
(0.95-1.10) \\
\end{array}$ & $<0.001 * * *$ \\
\hline
\end{tabular}

a: $\mathrm{p}$-value was calculated from independent samples T-test; SD: standard deviation; $* \mathrm{p}<0.05 ; * * \mathrm{p}<0.01, * * * \mathrm{p}<0.001$ 
Table (4): Backward elimination, multiple regression models for estimation of sex from hand and finger measurements

\begin{tabular}{|c|c|c|c|c|c|c|c|c|}
\hline Race & Side & $\begin{array}{l}\text { Nagelkerke } \\
\text { R Square }\end{array}$ & Parameters & B & SE & $\mathbf{p}$ & OR & $95 \%$ CI of OR \\
\hline \multirow{9}{*}{ Egyptians } & \multirow{3}{*}{ Right } & \multirow{3}{*}{0.595} & Constant & -8.50 & 3.82 & \begin{tabular}{|l|}
$0.026^{*}$ \\
\end{tabular} & & \\
\hline & & & Rt hand breadth (mm) & 0.50 & 0.07 & $<0.001^{* * * *}$ & 1.65 & 1.43 to 1.90 \\
\hline & & & Rt hand index & -0.71 & 0.15 & $<0.001^{* * *}$ & 0.49 & 0.36 to 0.66 \\
\hline & \multirow{6}{*}{ Left } & \multirow{6}{*}{0.623} & Constant & 148.88 & 95.27 & \begin{tabular}{|l|}
0.118 \\
\end{tabular} & & \\
\hline & & & Lt hand breadth $(\mathrm{mm})$ & 0.44 & 0.11 & $<0.001^{* * *}$ & 1.556 & 1.26 to 1.92 \\
\hline & & & Lt index length (mm) & 2.22 & 1.33 & $\begin{array}{l}0.094 \\
\end{array}$ & 9.183 & 0.68 to 123.24 \\
\hline & & & Lt ring length $(\mathrm{mm})$ & -2.13 & 1.30 & 0.102 & 0.119 & 0.01 to 1.53 \\
\hline & & & Lt hand index & -0.61 & 0.20 & $0.002^{* *}$ & 0.545 & 0.37 to 0.80 \\
\hline & & & Lt D2:D4 ratio & -164.10 & 96.91 & 0.090 & 0.000 & 0.00 to 166.73 \\
\hline \multirow{11}{*}{ Malaysians } & \multirow{6}{*}{ Right } & \multirow{6}{*}{0.816} & Constant & -120.51 & 65.35 & 0.065 & & \\
\hline & & & Rt hand length (mm) & 0.58 & 0.40 & 0.149 & 1.79 & 0.81 to 3.96 \\
\hline & & & Rt hand breadth (mm) & -0.73 & 0.83 & 0.383 & 0.48 & 0.10 to 2.47 \\
\hline & & & Rt index length $(\mathrm{mm})$ & -0.47 & 0.23 & $0.040^{*}$ & 0.63 & 0.40 to 0.98 \\
\hline & & & Rt ring length $(\mathrm{mm})$ & 0.49 & 0.24 & $0.041^{*}$ & 1.63 & 1.02 to 2.61 \\
\hline & & & Rt hand index & 1.65 & 1.39 & 0.237 & 5.20 & 0.34 to 79.64 \\
\hline & \multirow{5}{*}{ Left } & \multirow{5}{*}{0.841} & Constant & -88.58 & 22.05 & $<0.001^{* * * *}$ & & \\
\hline & & & Lt hand length (mm) & 0.26 & 0.09 & $0.002^{* *}$ & 1.30 & 1.10 to 1.54 \\
\hline & & & Lt index length $(\mathrm{mm})$ & -0.37 & 0.23 & 0.111 & 0.69 & 0.44 to 1.09 \\
\hline & & & Lt ring length $(\mathrm{mm})$ & 0.57 & 0.26 & $0.029^{*}$ & 1.77 & 1.06 to 2.97 \\
\hline & & & Lt hand index & 0.65 & 0.20 & $0.001^{* *}$ & 1.92 & 1.30 to 2.84 \\
\hline
\end{tabular}

B: regression coefficient; CI: confidence interval; SE: standard error; ${ }^{*} \mathrm{p}<0.05 ; * * \mathrm{p}<0.01,{ }^{* * *} \mathrm{p}<0.001$

Table (5): Spearman's rank-order correlation between stature and the studied hands and fingers measurements:

\begin{tabular}{|c|c|c|c|c|c|}
\hline & \multicolumn{4}{|c|}{ Stature (Cm) } \\
\hline & & \multicolumn{2}{|c|}{ Egyptians } & \multicolumn{2}{|c|}{ Malaysians } \\
\hline & & Male & Female & Male & Female \\
\hline \multirow{2}{*}{ Rt hand length (mm) } & $\mathrm{r}_{\mathrm{s}}$ & 0.715 & 0.573 & 0.520 & 0.484 \\
\hline & $\bar{p}$ & $<0.001^{* * * *}$ & $<0.001 * * *$ & $<0.001^{* * * *}$ & $<0.001^{* * * *}$ \\
\hline \multirow{2}{*}{ Rt hand breadth (mm) } & $\mathrm{r}_{\mathrm{S}}$ & 0.416 & 0.429 & 0.370 & 0.303 \\
\hline & $\mathrm{p}$ & $<0.001^{* * *}$ & $<0.001 * * *$ & $0.008^{* *}$ & $0.032^{*}$ \\
\hline \multirow{2}{*}{ Rt index length (mm) } & $\mathrm{r}_{\mathrm{S}}$ & 0.612 & 0.509 & 0.491 & 0.488 \\
\hline & $\mathrm{p}$ & $<0.001^{* * *}$ & $<0.001 * * *$ & $<0.001 * * *$ & $<0.001 * * *$ \\
\hline \multirow{2}{*}{ Rt ring length (mm) } & $\mathrm{r}_{\mathrm{s}}$ & 0.496 & 0.447 & 0.479 & 0.450 \\
\hline & $\mathrm{p}$ & $<0.001^{* * *}$ & $<0.001 * * *$ & $<0.001 * * *$ & $<0.001 * * *$ \\
\hline \multirow{2}{*}{ Rt hand index } & $\mathrm{r}_{\mathrm{S}}$ & -0.034 & -0.007 & 0.019 & -0.278 \\
\hline & $\mathrm{p}$ & 0.743 & 0.943 & 0.898 & 0.051 \\
\hline \multirow{2}{*}{ Rt D2:D4 ratio } & $\mathrm{r}_{\mathrm{s}}$ & 0.010 & -0.042 & -0.023 & 0.106 \\
\hline & $\mathrm{p}$ & 0.923 & 0.674 & 0.872 & 0.463 \\
\hline \multirow{2}{*}{ Lt hand length (mm) } & $\mathrm{r}_{\mathrm{s}}$ & 0.700 & 0.643 & 0.512 & 0.529 \\
\hline & $\mathrm{p}$ & $<0.001 * * *$ & $<0.001 * * *$ & $<0.001 * * *$ & $<0.001 * * *$ \\
\hline \multirow{2}{*}{ Lt hand breadth (mm) } & $\mathrm{r}_{\mathrm{s}}$ & 0.433 & 0.440 & 0.456 & 0.378 \\
\hline & $\mathrm{p}$ & $<0.001 * * *$ & $<0.001 * * *$ & $0.001 * *$ & $0.007 * *$ \\
\hline \multirow{2}{*}{ Lt index length (mm) } & $\mathrm{r}_{\mathrm{s}}$ & 0.597 & 0.514 & 0.471 & 0.472 \\
\hline & $\mathrm{p}$ & $<0.001^{* * *}$ & $<0.001 * * *$ & $0.001^{* *}$ & $0.001^{* *}$ \\
\hline \multirow{2}{*}{ Lt ring length (mm) } & $\mathrm{r}_{\mathrm{S}}$ & 0.646 & 0.437 & 0.410 & 0.397 \\
\hline & $\mathrm{p}$ & $<0.001^{* * *}$ & $<0.001 * * *$ & $0.003^{* *}$ & $0.004^{* *}$ \\
\hline \multirow{2}{*}{ Lt hand index } & $\mathrm{r}_{\mathrm{s}}$ & -0.087 & -0.017 & 0.007 & -0.031 \\
\hline & $\mathrm{p}$ & 0.399 & 0.863 & 0.959 & 0.833 \\
\hline \multirow{2}{*}{ Lt D2:D4 ratio } & $\mathrm{r}_{\mathrm{s}}$ & 0.036 & -0.041 & -0.022 & 0.102 \\
\hline & $\mathrm{p}$ & 0.725 & 0.682 & 0.878 & 0.481 \\
\hline
\end{tabular}

$\mathrm{r}_{\mathrm{s}}$ : correlation coefficient; ${ }^{*} \mathrm{p}<0.05 ; * * \mathrm{p}<0.01,{ }^{* * *} \mathrm{p}<0.001$ 
Table (6): Backward elimination, multiple regression models for estimation of stature from hand and finger measurements

\begin{tabular}{|c|c|c|c|c|c|c|c|}
\hline Race & Side & $\begin{array}{c}\text { Adjusted R } \\
\text { square }\end{array}$ & Parameters & B & SE & $\mathbf{p}$ & $95 \%$ CI of B \\
\hline \multirow{7}{*}{ Egyptians } & \multirow{4}{*}{ Right } & \multirow{4}{*}{0.733} & Constant & 79.02 & 7.14 & $<0.001 * * *$ & 64.94 to 93.09 \\
\hline & & & Rt hand length (mm) & 0.38 & 0.07 & $<0.001^{* * *}$ & 0.24 to 0.52 \\
\hline & & & Rt ring length $(\mathrm{mm})$ & 0.24 & 0.13 & 0.058 & -0.01 to 0.50 \\
\hline & & & Sex & 5.93 & 0.89 & $<0.001^{* * *}$ & 4.17 to 7.68 \\
\hline & \multirow{3}{*}{ Left } & \multirow{3}{*}{0.748} & Constant & 71.93 & 6.87 & $<0.001^{* * *}$ & 58.39 to 85.48 \\
\hline & & & Lt hand length $(\mathrm{mm})$ & 0.52 & 0.04 & $<0.001^{* * *}$ & 0.44 to 0.59 \\
\hline & & & Sex & 5.60 & 0.87 & $<0.001^{* * *}$ & 3.89 to 7.32 \\
\hline \multirow{10}{*}{ Malaysians } & \multirow{5}{*}{ Right } & \multirow{5}{*}{0.543} & Constant & -56.6 & 51.70 & 0.277 & -159.21 to 46.07 \\
\hline & & & Rt hand length $(\mathrm{mm})$ & 1.18 & 0.32 & $<0.001^{* * *}$ & 0.54 to 1.82 \\
\hline & & & Rt hand breadth $(\mathrm{mm})$ & -1.82 & 0.67 & $0.008^{* *}$ & -3.15 to -0.49 \\
\hline & & & Rt hand index & 3.46 & 1.03 & $0.001^{* *}$ & 1.41 to 5.51 \\
\hline & & & Sex & 3.30 & 1.89 & 0.084 & -0.45 to 7.06 \\
\hline & \multirow{5}{*}{ Left } & \multirow{5}{*}{0.550} & Constant & -71.87 & 50.97 & $0.016^{* *}$ & -173.06 to 29.32 \\
\hline & & & Lt hand length $(\mathrm{mm})$ & 1.30 & 0.32 & $<0.001^{* * *}$ & 0.67 to 1.93 \\
\hline & & & Lt hand breadth $(\mathrm{mm})$ & -2.12 & 0.66 & $0.002^{* *}$ & -3.43 to -0.81 \\
\hline & & & Lt hand index & 3.87 & 1.02 & $<0.001^{* * *}$ & 1.84 to 5.89 \\
\hline & & & Sex & 3.43 & 1.86 & 0.068 & -0.26 to 7.11 \\
\hline
\end{tabular}

B: regression coefficient; CI: confidence interval; SE: standard error; ${ }^{*} \mathrm{p}<0.05 ; * * \mathrm{p}<0.01,{ }^{* * *} \mathrm{p}<0.001$
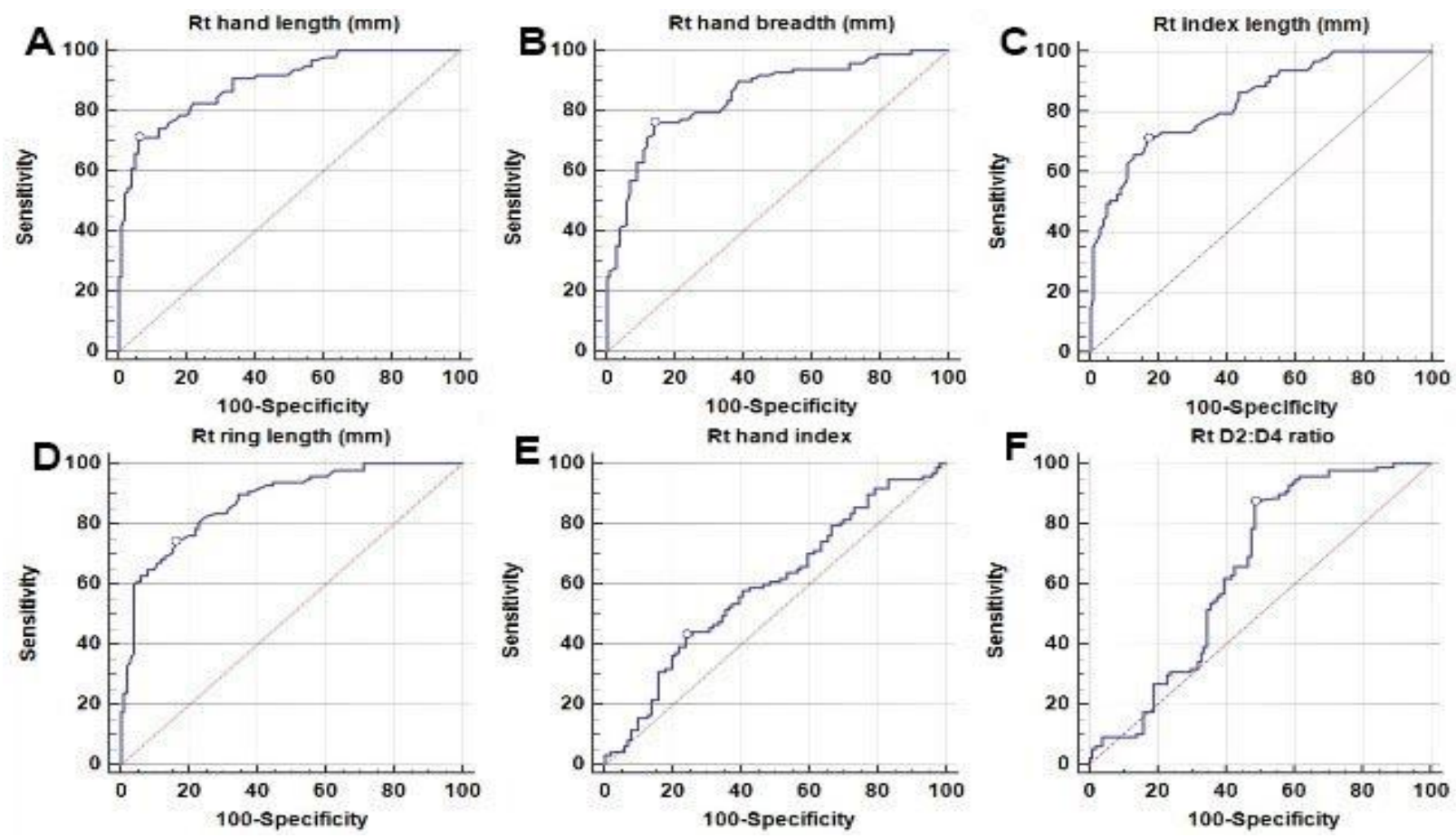

Fig. (2): ROC curves for prediction of male sex in Egyptian participants using: (A) right hand length $\left(\right.$ AUC $=0.892, \mathrm{p}<0.001^{* * *}$, cut-off $>183.6 \mathrm{~mm}$, sensitivity $=71.1 \%$, specificity $\left.=94.1 \%\right)$; (B) right hand breadth $\left(\mathrm{AUC}=0.852, \mathrm{p}<0.001^{* * *}\right.$, cut-off $>81.5 \mathrm{~mm}$, sensitivity $=76.3 \%$, specificity $=86.1 \%) ;(\mathrm{C})$ right index finger length $\left(\mathrm{AUC}=0.834, \mathrm{p}<0.001^{* * *}\right.$, cut-off $>70.5 \mathrm{~mm}$, sensitivity $=71.1 \%$, specificity $=83.2 \%)$; (D) right ring finger length $(\mathrm{AUC}=0.875, \mathrm{p}<0.001 * * *$, cut-off $>71.7 \mathrm{~mm}$, sensitivity $=74.2 \%$, specificity $=84.2 \%$; $(\mathrm{E})$ right hand index $(\mathrm{AUC}=0.595$, $\mathrm{p}=<0.021^{* *}$, cut-off $>45.8$, sensitivity $=43.3 \%$, specificity $\left.=76.2 \%\right)$; and (F) right D2:D4 ratio $\left(\mathrm{AUC}=0.649, \mathrm{p}<0.001^{* * *}\right.$, cut-off $\leq 0.9988$, sensitivity $=87.6 \%$, specificity $\left.=51.5 \%\right)$. 

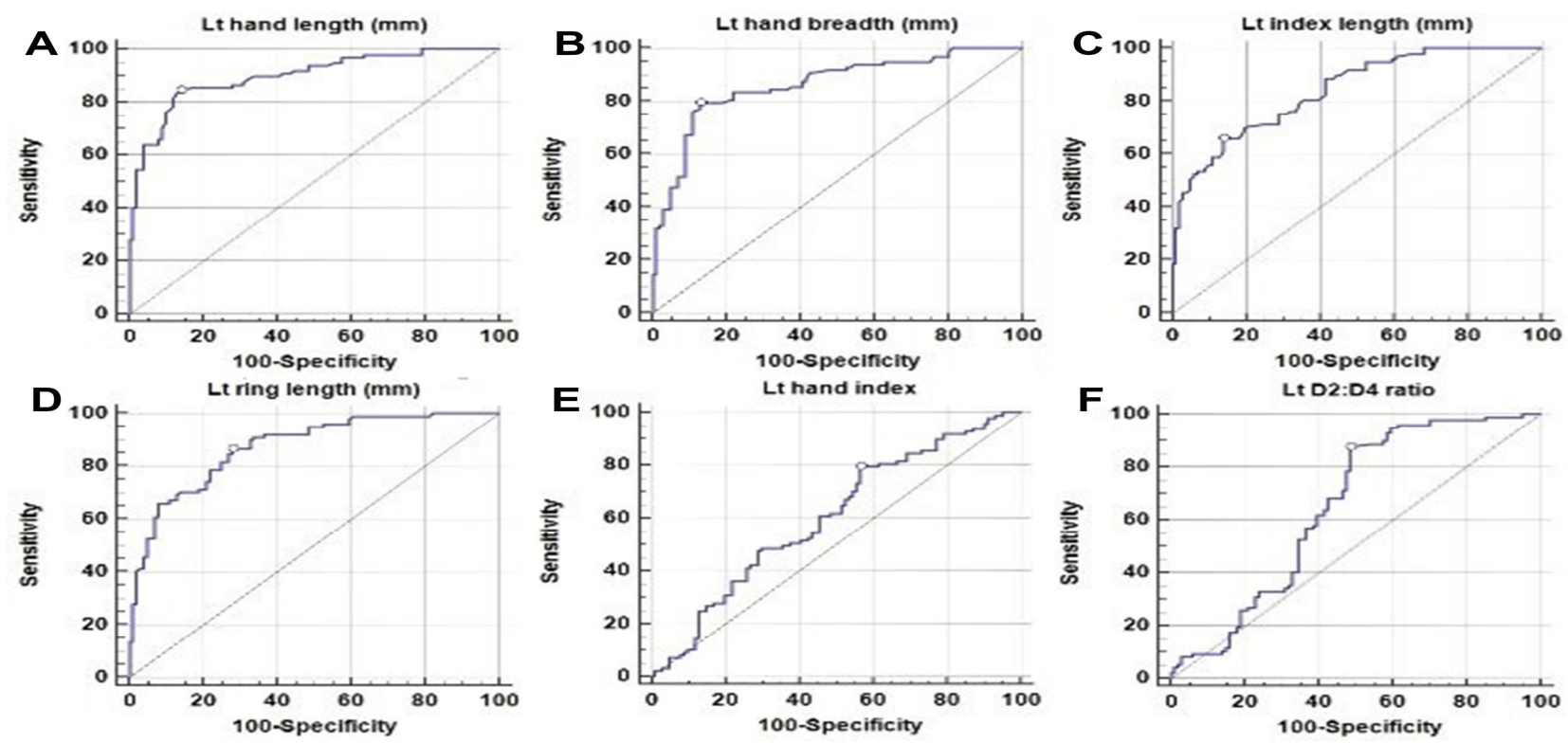

Fig. (3): ROC curves for prediction of male sex in Egyptian participants using: (A) left hand length (AUC $=0.898, \mathrm{p}<0.001^{* * *}$, cut-off $>181.5 \mathrm{~mm}$, sensitivity $=84.5 \%$, specificity $=86.1 \%$ ); (B) left hand breadth (AUC $=0.862, \mathrm{p}<0.001 * * *$, cut-off $>81.3 \mathrm{~mm}$, sensitivity $=79.4 \%$, specificity $=87.1 \%$ ); (C) left index finger length (AUC $=0.839, \mathrm{p}<0.001^{* * *}$, cut-off $>71.4 \mathrm{~mm}$, sensitivity $=66.0 \%$, specificity $=86.1 \%$ ); (D) left ring finger length (AUC $=0.872, \mathrm{p}<0.001^{* * *}$, cut-off $>70.7 \mathrm{~mm}$, sensitivity $=86.6 \%$, specificity $=72.3 \%)$; (E) left hand index $\left(\mathrm{AUC}=0.602, \mathrm{p}=0.013^{* *}\right.$, cut-off $>43.2$, sensitivity $=79.4 \%$, specificity $=43.6 \%$ ); and $(\mathrm{F})$ left $\mathrm{D} 2 \mathrm{:D}$ 4 ratio $(\mathrm{AUC}=0.651$, $\mathrm{p}<0.001^{* * *}$, cut-off $\leq 0.9988$, sensitivity $=87.6 \%$, specificity $=51.5 \%$ ).
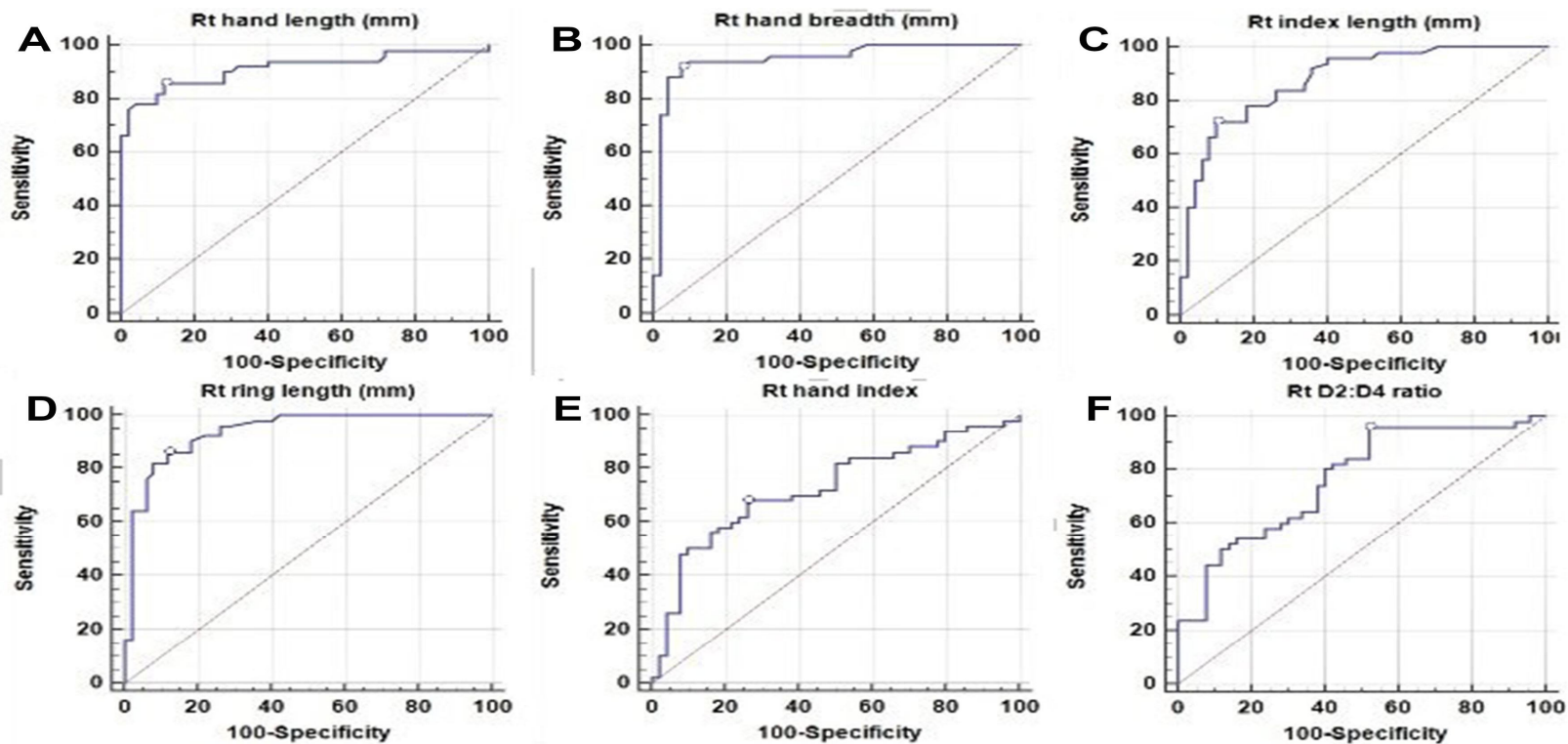

Fig. (4): ROC curves for prediction of male sex in Malaysian participants using: (A) right hand length (AUC $=0.915, \mathrm{p}<0.001^{* * *}$, cut-off $>173.1 \mathrm{~mm}$, sensitivity $=86.0 \%$, specificity $=88.0 \%$ ); (B) right hand breadth $\left(\mathrm{AUC}=0.949, \mathrm{p}<0.001^{* * *}\right.$, cut-off $>76.2 \mathrm{~mm}$, sensitivity $=92.0 \%$, specificity $=92.0 \%) ;(\mathrm{C})$ right index finger length $\left(\mathrm{AUC}=0.880, \mathrm{p}<0.001^{* * *}\right.$, cut-off $>68.7 \mathrm{~mm}$, sensitivity $=72.0 \%$, specificity $=90.0 \%)$; (D) right ring finger length $\left(\mathrm{AUC}=0.938, \mathrm{p}<0.001^{* * *}\right.$, cut-off $>68.2 \mathrm{~mm}$, sensitivity $=86.0 \%$, specificity $=88.0 \%)$; $(\mathrm{E})$ right hand index $(\mathrm{AUC}=0.726$, $\mathrm{p}<0.001^{* * *}$, cut-off $>44.4$, sensitivity $=68.0 \%$, specificity $\left.=74.0 \%\right)$; and $(\mathrm{F})$ right $\mathrm{D} 2: \mathrm{D} 4$ ratio $\left(\mathrm{AUC}=0.763, \mathrm{p}<0.001^{* * *}\right.$, cut-off $\leq 1.0015$, sensitivity $=96.0 \%$, specificity $\left.=48.0 \%\right)$. 

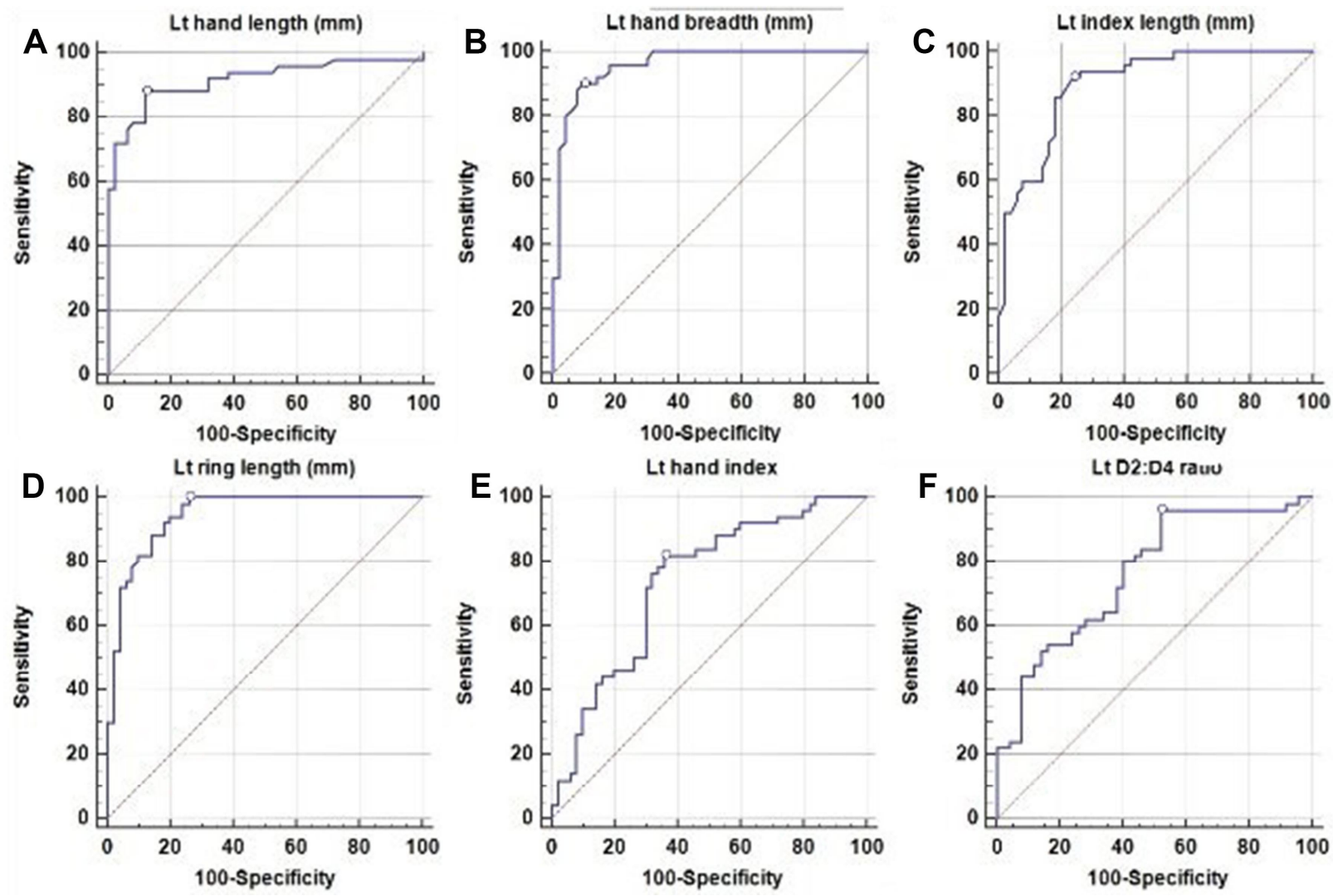

Fig. (5): ROC curves for prediction of male sex in Malaysian participants using: (A) left hand length (AUC $=0.916, \mathrm{p}<0.001^{* * *}$, cut-off $>172.6 \mathrm{~mm}$, sensitivity $=88.0 \%$, specificity $=88.0 \%$ ); (B) left hand breadth (AUC $=0.959, \mathrm{p}<0.001 * * *$, cut-off $>77.1 \mathrm{~mm}$, sensitivity $=90.0 \%$, specificity $=90.0 \%$ ); (C) left index finger length (AUC $=0.899, \mathrm{p}<0.001 * * *$, cut-off $>67.3 \mathrm{~mm}$, sensitivity $=92.0 \%$, specificity $=76.0 \%$ ); (D) left ring finger length (AUC $=$ $0.945, \mathrm{p}<0.001^{* * *}$, cut-off $>66.4 \mathrm{~mm}$, sensitivity $=100.0 \%$, specificity $=74.0 \%$ ); (E) left hand index (AUC $=0.734, \mathrm{p}<0.001^{* * *}$, cut-off $>43.3$, sensitivity $=82.0 \%$, specificity $=$ $64.0 \%$ ); and $(\mathrm{F})$ left $\mathrm{D} 2: \mathrm{D} 4$ ratio $\left(\mathrm{AUC}=0.762, \mathrm{p}<0.001^{* * *}\right.$, cut-off $\leq 1.0014$, sensitivity $=$ $96.0 \%$, specificity $=48.0 \%$ ).

\section{Discussion}

The present study was conducted to evaluate hand indices as a potential tool for determination of sex and estimation of stature in a sample of Egyptian and Malaysian people. This study was carried out on 200 Egyptian and 100 Malaysian volunteers.

Malaysian subjects were included in this study to assess variability in hand dimensions between two populations of different ethnic backgrounds. Moreover, Malaysian subjects visit Egypt and reside for varying lengths of time for educational and recreational purposes, therefore they may be involved in investigations of mass disasters or identification of unknown remains in accidents taking place in Egypt.

The age of the participants in this study was above 21 years and less than 30 years old. This age group was selected in the present study to ensure complete development, growth, and maturity of the included subjects. The union of metacarpal and phalangeal epiphyses occurs between the age of 14 and 17 years in males in non-tropical climates; the union occurs slightly earlier in females (Knight, 1997). Consequently, the lower limit of age for 
participants was determined to be 21 years old. As for the upper limit of 30 years, this age was selected to avoid age-related changes in stature. After the age of 25 years, human height becomes shortened by approximately $2.5 \mathrm{~cm}$ for every 25 years (Vij, 2011). Some studies suggest that height changes commence at the age of 40 years (Krishan et al., 2012).

The mean stature was higher in male Egyptian participants $(174.9 \pm 7.2 \mathrm{~cm})$ and Malaysian participants $(169.1 \pm 6.3 \mathrm{~cm})$ compared to female Egyptian (161.7 \pm 5.2 $\mathrm{cm})$ and Malaysian subjects $(158.8 \pm 5.8 \mathrm{~cm})$. The mean values of stature in the present study were similar to those reported in both Egyptian (Habib and Kamal, 2010; Sharaf Eldin et al., 2015) and Malaysian populations (Nor et al., 2013).

Comparison of measurements between right and left hands and fingers revealed the lack of significant difference between rightand left-side measurements within all categories of participants. This finding was in accordance with previous studies which found non-significant, trivial differences between hand dimensions of right and left sides (Krishan and Sharma, 2007; Habib and Kamal, 2010; Aboul-Hagag et al., 2011).

The mean values of hand length and breadth in male and female Egyptian participants in the present study were slightly longer than those reported in Indian (Krishan and Sharma, 2007; Varu et al., 2015), Malaysian (Jeyaseelan et al., 2016), and Turkish (Kulaksiz and Gözil, 2002) populations. Meanwhile, the mean length of right and left hands in our Egyptian participants was shorter than those reported in other studies on Egyptian in Upper Egypt (Habib and Kamal, 2010; Aboul-Hagag et al., 2011), Indian (Kanchan and Rastogi, 2009), and Nigerian (Danborno and Elukpo, 2007) populations. These differences in measurements among Egyptian studies may be related to inter-observer variability or to variations in the Egyptian subpopulations as the two studies were conducted in upper Egypt. Even within the same country, variations were reported from one region to another due to different mixing of ethnic origins, climate and lifestyle, as observed particularly in studies from India. The mean values of hand length and breadth in male and female Malaysian participants were comparable to those reported in a previous study on Malaysian population (Jeyaseelan et al., 2016).

The hand index among our participants was slightly higher than reported by Kanchan and Rastogi (2009) and Aboul-Hagag et al.(2011), as our participants had shorter hand length with nearly similar hand breadth to those reported by them.

These variations among the different populations in hand measurements were observed before in the literature (Okunribido, 2000; Aboul-Hagag et al., 2011; Swami et al., 2013) and could be explained by differences in genetic constitution and environmental effects such as the climate and nutritional factors (Silventoinen et al., 2007). This variation also among populations highlights the need of conducting forensic anthropometric studies for each population separately and warns against the application of equations derived from one population to another one.

Comparison of the measurements of both right and left hands and fingers between the studied male and female subjects yielded significant differences. All measurements were higher in male than female subjects in the Egyptian and Malaysian samples, except for the D2:D4 ratio which was significantly higher in female than in male subjects. These significant differences indicated that hand measurements could potentially be used to discriminate between male and female subjects.

The D2:D4 ratio has been studied in both sexes. The index and ring finger are reported to 
be nearly of the same length in females, while the ring finger is usually longer than the index finger in males. This observation suggests the potential use of D2:D4 ratio in identification of sex (Kanchan et al., 2008; Kanchan et al., 2010). The D2:D4 ratio was found to be present early in neonates and remains stable thereafter during life (Manning et al., 2004).

These significant differences between men and women of the same population agree with previous reports in different human populations including Egyptians (Habib and Kamal, 2010; Aboul-Hagag et al., 2011), Saudis (Ibrahim et al., 2016), Indians (Jasuja and Singh, 2004; Agnihotri et al., 2006; Krishan, 2007; Kanchan et al., 2008; Kanchan and Rastogi, 2009; Kanchan et al., 2010), and Malaysians (Jeyaseelan et al., 2016). These statistically significant differences could be explained by the fact that girls reach puberty earlier than boys; therefore, boys obtain two more years of physical growth than girls (Krishan, 2007).

The present study evaluated the discriminatory power for identification of sex of the studied hand measurements using ROC curve analysis. In Egyptian participants, good predictors of sex were hand length, followed by ring finger length, hand breadth, and index finger length, as their AUC was above 0.8. On the other hand, the D2:D4 ratio and hand index had much lower discriminatory power, as their AUCs ranged from 0.6 to less than 0.7 .

In the Malaysian participants, good to excellent predictors of sex were identified including hand breadth, ring finger length, hand length, and index finger length, as the AUCs of these measurements were all above 0.8 . The D2:D4 ratio and hand index were fair predictors as their AUCs ranged from 0.7 to less than 0.8 .

These results are in partial agreement with similar previous studies. Krishan et al.
(2011) stated that hand breadth and hand length were good predictors for sex in a north Indian population. Kanchan and Rastogi (2009) suggested that hand breadth was the most reliable sex discriminator in their study on North and South Indians. Ishak and colleagues (2012) reported that hand breadth and length showed an accuracy of $94 \%$ and $91.5 \%$, respectively in West Australian subjects. On the other hand, Jee and colleagues (2015) reported only an accuracy of $4.2 \%$ for sex discrimination in a Korean population. Ibrahim et al.(2016) evaluated hand index and D2:D4 ratio in a Saudi adult populations, reporting much higher AUC for both indices (AUC > 0.8 ). This may indicate that the performance of hand dimensions as discriminators of sex varies widely from one population to another. Consequently, there is a need to identify the best performing discriminators in each population and to develop population-specific equations for estimations of sex based on the comparison among the potential discriminators.

Although it has been hypothesized that ratios between hand dimensions would provide more accurate estimation of sex than the original, separate measurements of hands and fingers (Lippa, 2003), our study results suggest that the performance of hand index and D2:D4 ratio as discriminators of sex is inferior to hand length, hand breadth, index finger length, and ring finger length. Opponents of hand and finger ratios suggest that these indices are not directly related to physical body built or age (Lippa, 2003). Most previous studies have not compared hand index and D2:D4 ratios to other hand measurements, and several studies did not use regression, discriminant function analysis, or ROC curve analysis to assess the performance of these indices in discrimination of sex. Instead, many studies relied on comparison with T-test or similar nonparametric equivalent and cut-off values were arbitrarily calculated. The value of D2:D4 ratio as a discriminator of sex was disputed also by 
Kosif and Diramal1(2012) who reported the lack of significant difference between men and women. Moreover, Camacho-Hernández et al.(2018) reported a relatively low accuracy for D2:D4 ratio in discrimination of sex (around 60\%, preceded by other finger ratios) and undermined its value on their study on a population from Canary island. However, the present study evaluated hand dimensions within a restricted age group. The value of hand index and D2:D4 ratio may be greater in other age groups or in a population with heterogeneous age as these dimensions are not affected by age or growth.

In the current study, the correlations between the studied measurements and stature were assessed to identify potential measurements which could be used to calculate stature. There was a positive, significant, moderate to strong correlation between stature and each of hand length, hand breadth, index finger length, and ring finger length. These findings indicated that the mentioned measurements could be used as a potential tool to calculate stature. On the other hand, the correlation between stature and each of hand index and D2:D4 ratio was weak and non-significant, indicating that either measurement would not be valid for estimation of stature if used alone.

Our findings are in accordance with other studies which reported similar significant correlations (Jasuja and Singh, 2004; Krishan, 2007; Rastogi et al., 2008; Ahmad et al., 2014). Meanwhile, Suseelamma et al. (2014) concluded that ring finger was the best indicator of height in their population of Indian subjects, however, they did not evaluate hand length, hand breadth, or hand index for calculation of stature.

As stature correlated significantly with many hand dimensions, the present study proceeded then to derive models for estimation of stature from the studied measurements. Equations for stature estimation in Egyptians and Malaysians were developed.

The value of hand measurements in estimation of stature was reported before in previous studies (Agnihotri et al., 2008; Ahmed, 2013; Rhiu and Kim, 2019), with varying equations which utilized a combination of different hand dimensions. Comparison of our equations for sex or stature with those of previous studies is not applicable as we stated before that measurements vary widely across different populations.

\section{Conclusion and recommendations}

Hand dimensions can serve as a tool to predict sex and calculate stature with reasonable accuracy in Egyptian and Malaysian youths. The advantages of using hand dimensions in identification of sex and estimation of stature are numerous as this method is simple, easy to perform, and not costly. Using hand dimensions can be regarded as a screening method in the identification process, obviating the use of sophisticated expensive methods and equipment during the initial stages of identification. Nevertheless, the equations derived in the present study need further validation in future studies with larger sample sizes. Future studies should preferably include Egyptian and Malaysian subjects from various areas to represent all potential variations within both populations. Testing the derived cut-off values and equations on older age groups is also recommended.

\section{Conflict of interest}

All authors of the current research declared no conflict of interest. 


\section{References}

Aboul-Hagag, K.E.; Mohamed, S.A.; Hilal, M.A.; et al. (2011): "Determination of sex from hand dimensions and index/ring finger length ratio in Upper Egyptians". Egypt J. Forensic Sci., 1 (2): 80-86.

Agnihotri, A.; Purwar, B.; Jeebun, N.; et al. (2006): "Determination of sex by hand dimensions". The Internet Journal of Forensic Science, 1 (2): 12-24.

Agnihotri, A.K.; Agnihotri, S.; Jeebun, N.; et al. (2008): "Prediction of stature using hand dimensions". J. Forensic Leg. Med., 15 (8): 479-482.

Ahmad, N.; Mohammad, F. and Farooque, I. (2014): "Estimation of height from the long bones of upper limb and hand dimensions in South Indian population". J. Evidence Based Med. Health Care, 1 (7): 473-478.

Ahmed, A.A. (2013): "Estimation of stature from the upper limb measurements of Sudanese adults". Forensic Sci. Int., 228 (1-3): 178. e171-178. e177.

Camacho-Hernández, C.M.; PerdigónAlvarado, E.; Quintero-Platt, G.; et al. (2018): "Digit ratios among the modern population of the Canary Islands". Eur. J. Anat., 22 (2): 145-155.

Danborno, B. and Elukpo, A. (2007): "Sexual dimorphism in hand and foot length, indices, stature-ratio and relationship to height in Nigerians". The Internet Journal of Forensic Science, 3 (1).

DeLong, E.R.; DeLong, D.M. and ClarkePearson, D.L. (1988): "Comparing the areas under two or more correlated receiver operating characteristic curves: a nonparametric approach". Biometrics, 44 (3): 837-845.
Habib, S.R. and Kamal, N.N. (2010): "Stature estimation from hand and phalanges lengths of Egyptians". J. Forensic Leg. Med., 17 (3): 156-160.

Hrycaj, S.M. and Wellik, D.M. (2016): "Hox genes and evolution". F1000Res, 5: F1000 Faculty Rev-1859.

Ibrahim, M.A.; Khalifa, A.M.; Hagras, A.M.; et al. (2016): "Sex determination from hand dimensions and index/ring finger length ratio in North Saudi population: Medico-legal view". Egypt J. Forensic Sci., 6 (4): 435-444.

Ishak, N.-I.; Hemy, N. and Franklin, D. (2012): "Estimation of sex from hand and handprint dimensions in a Western Australian population". Forensic Sci. Int., 221 (1-3): 154. e151-154. e156.

Jasuja, O.P. and Singh (2004): "Estimation of stature from hand and phalange length". J. Indian Acad. Forensic Med., 26: 100 106.

Jee, S.C.; Bahn, S. and Yun, M.H. (2015): "Determination of sex from various hand dimensions of Koreans". Forensic Sci. Int., 257: 521.e521-521.e510.

Jeyaseelan, N.; Ravindran, P.; Pitadeniya, U.M.; et al. (2016): "Estimation of hand index and sex variations among the university students of Malaysia-An anthropometric study". J. Humanities Social Sci.,21: 32-37.

Kanchan, T.; Krishan, K.; Sharma, A.; et al. (2010): "A study of correlation of hand and foot dimensions for personal identification in mass disasters". Forensic Sci. Int., 199 (1-3): 112.e111-116.

Kanchan, T.; Kumar, G.P. and Menezes, R.G. (2008): "Index and ring finger ratio-a new sex determinant in south Indian population". Forensic Sci. Int., 181 (1-3): 53.e51-54. 
Kanchan, T. and Rastogi, P. (2009): "Sex determination from hand dimensions of North and South Indians". J. Forensic Sci., 54 (3): 546-550.

Knight, B. (1997): The establishment of identity of human remains. In: Forensic pathology. Second ed, Arnold Publishing Ltd., London, Great Britain, P.P. 119.

Kosif, R. and Diramalı, M. (2012): "Comparison of all hand digit length ratios in left - and righthanded individuals". Turk. J. Med. Sci.,42: 545552.

Krishan, K. (2007): "Anthropometry in forensic medicine and forensic scienceForensic Anthropometry". Internet Journal of Forensic Sciences, 2 (1).

Krishan, K.; Kanchan, T.; Menezes, R.G.; et al. (2012): "Forensic anthropology casework - essential methodological considerations in stature estimation". J. Forensic Nurs., 8 (1): 45-50.

Krishan, K.; Kanchan, T. and Sharma, A. (2011): "Sex determination from hand and foot dimensions in a North Indian population". J. Forensic Sci.,56 (2): 453-459.

Krishan, K. and Sharma, A. (2007): "Estimation of stature from dimensions of hands and feet in a North Indian population". J. Forensic Leg. Med., 14 (6): 327-332.

Kulaksiz, G. and Gözil, R. (2002): "The effect of hand preference on hand anthropometric measurements in healthy individuals". Ann. Anat., 184 (3): 257265.

Lippa, R.A. (2003): “Are 2D: 4D fingerlength ratios related to sexual orientation? Yes for men, no for women". J. Pers. Soc. Psychol., 85 (1): 179.
Looker, A.C.; Melton, L.J., 3rd; Harris, T.; et al. (2009): "Age, gender, and race/ethnic differences in total body and subregional bone density". Osteoporos. Int., 20 (7): 1141-1149.

Manning, J.T.; Stewart, A.; Bundred, P.E.; et al. (2004): "Sex and ethnic differences in 2 nd to 4th digit ratio of children". Early Hum. Dev., 80 (2): 161-168.

Nor, F.M.; Abdullah, N.; Mustapa, A.-M.; et al. (2013): "Estimation of stature by using lower limb dimensions in the Malaysian population". J. Forensic Leg. Med., 20 (8): 947-952.

Okunribido, O.O. (2000): "A survey of hand anthropometry of female rural farm workers in Ibadan, Western Nigeria". Ergonomics, 43 (2): 282-292.

Rastogi, P.; Nagesh, K. and Yoganarasimha, K. (2008): "Estimation of stature from hand dimensions of north and south Indians". Leg. Med., 10 (4): 185-189.

Rhiu, I. and Kim, W. (2019): "Estimation of stature from finger and phalange lengths in a Korean adolescent". J. Physiol. Anthropol,38 (1): 13.

Sharaf Eldin, A.; Elkholy, S. and Hasan, M. (2015): "Prediction of Stature Based on Upper Limb Measurements among Egyptian Population". Eur. J. Forensic Sci., 3 (2): 1-7.

Silventoinen, K.; Bartels, M.; Posthuma, D.; et al. (2007): "Genetic regulation of growth in height and weight from 3 to 12 years of age: a longitudinal study of Dutch twin children". Twin Res. Hum. Genet., 10 (2): 354-363.

Suseelamma, D.; Gayathri, P.; Deepthi, S.; et al. (2014): "Study of Correlation between Stature and Length of Fingers". Sch. J. App. Med. Sci.,2 (2(2D)): 773784. 
Swami, S.; Kumar, T.; Sharma, D.; et al. (2013): "Effect of hand preference on second to fourth digit ratio and its role in sexual dimorphism: A study in 300 Haryanvi brahmins and 300 Kashmiri Pandits". Eur. J. Anat., 17: 243-249.

Varu, P.R.; Manvar, P.J.; Mangal, H.; et al. (2015): "Determination of stature from hand dimensions". J. Med. Res., 1 (3): 104-107.

Ventura, T.; Gomes, M.C.; Pita, A.; et al. (2013): "Digit ratio $(2 \mathrm{D}: 4 \mathrm{D})$ in newborns: influences of prenatal testosterone and maternal environment". Early Hum. Dev., 89 (2): 107-112.
Vij, K. (2011): Identification. In: Textbook of Forensic Medicine and Toxicology: Principles and Practice. 5th ed, Elsevier, India, P.P. 51.

Warrington, N.M.; Shevroja, E.; Hemani, G.; et al. (2018): "Genome-wide association study identifies nine novel loci for 2D:4D finger ratio, a putative retrospective biomarker of testosterone exposure in utero". Hum. Mol. Genet., 27 (11): 2025-2038.

Weiner, J.S. and Laurie, J.A. (1969): Human Biology-A Guide to Field Methods. Blackwell scientific publications, London. 


\title{
دراسة مؤشرات اليد و الأصابع للتنبؤ بالنوع و تقدير طول القامة

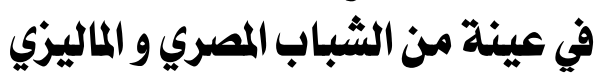

\author{
أمل سعيل أحمد فتحي و مروة محمد شاهين

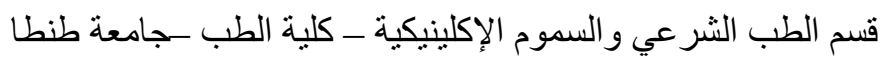

يعد الإستعر اف حجر الزاوية في التحقيقات الطبية الثرعية. هدفت الدراسة الحالية إلى تقييم مؤشرات اليد

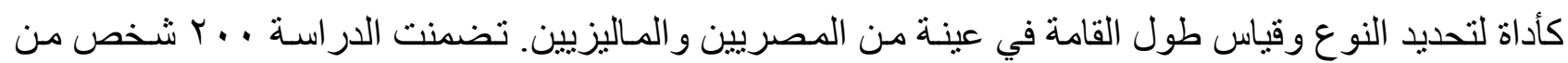

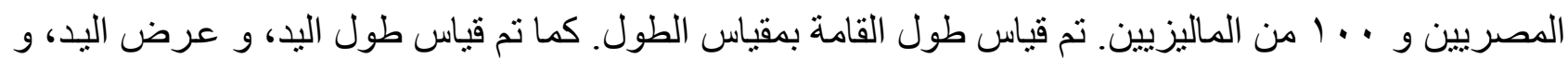
طول إصبع السبابة، و طول إصبع الخنصر بمقياس فيرنيـه، تم حساب مؤشر اليد كالأتي: (عرض اليد/ طول

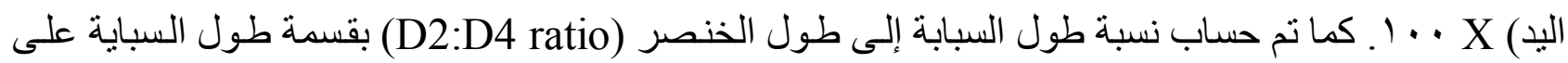
طول الخنصر في البد الواحدة. جميع القياسات كانت أعلى في الذكور عن الإناث بفرق ذي دلالة إحصائية، فيما عدا نسبة طول السبابة إلى الخنصر كانت أعلى في الإناث عن الذكور بفرق ذبي دلالة إحصائية في المصريين

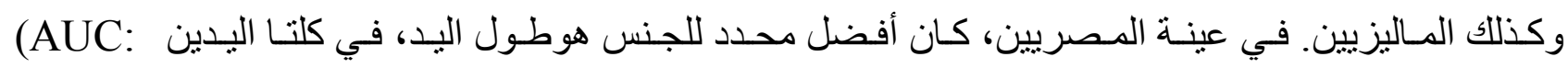
Rt=0.892, Lt: AUC=0.898) اليدين (Rt: AUC=0.949, Lt: AUC=0.959). في جميع المجمو عات، كان هناك ارتباط إيجابي ذو دلالة إحصائية منوسطة إلى قوية بين طول القامة وكلا من طول اليد، و عرض اليد، و طول السبابة، وطول الخنصر.

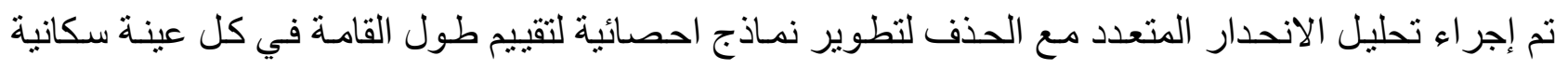
باستخدام قياسات من يد واحدة. وبذلك نستنتج أن قياسات اليد يمكن أن تستخدم كأداة لتوقع النوع وحساب طول القامة بدقة معقولة في الثباب المصريين و الماليزيين. 